\title{
Perturbations and chaos in quantum maps
}

\author{
Darío E. Bullo ${ }^{1}$ and Diego A. Wisniacki ${ }^{1, *}$ \\ ${ }^{1}$ Departamento de Fúsica "J. J. Giambiagi", FCEN, Universidad de Buenos Aires, 1428 Buenos Aires, Argentina
}

(Dated: July 21, 2018)

\begin{abstract}
The local density of states (LDOS) is a distribution that characterizes the effect of perturbations on quantum systems. Recently, it was proposed a semiclassical theory for the LDOS of chaotic billiards and maps. This theory predicts that the LDOS is a Breit-Wigner distribution independent of the perturbation strength and also gives a semiclassical expression for the LDOS witdth. Here, we test the validity of such an approximation in quantum maps varying the degree of chaoticity, the region in phase space where the perturbation is applying and the intensity of the perturbation. We show that for highly chaotic maps or strong perturbations the semiclassical theory of the LDOS is accurate to describe the quantum distribution. Moreover, the width of the LDOS is also well represented for its semiclassical expression in the case of mixed classical dynamics.
\end{abstract}

PACS numbers: 05.45.Mt; 05.45.Ac; 05.45.Pq

\section{INTRODUCTION}

The response of quantum systems to external perturbations is a problem of paramount importance in many areas of physics. Many of the properties of complex quantum system change dramatically when the system is perturbed, generating fundamental phenomena as quantum phase transitions, irreversibility or dissipation. The present development of experimental technics in quantum complex systems make the understanding and characterization of the effect of perturbations highly desirable.

The most likely suitable magnitude to characterize the effects of perturbations on quantum systems is the local density of states (LDOS). The LDOS, also called strength function, was introduced by Wigner [1] to understand the statistical properties of the wave functions of complex quantum systems. The LDOS is the profile of an eigenstate of an unperturbed quantum system over the eigenbasis of its perturbed version. To be more specific, let us consider a system with a one parameter dependent Hamiltonian $H(k)$ with eigenfrecuencies $\omega_{j}(k)$ and eigenstates $\left|\psi_{j}(k)\right\rangle$. The LDOS of an eigenstate $\left|\psi_{i}\left(k_{0}\right)\right\rangle$ (that we call unperturbed) is given by

$$
\rho_{i}(\omega, \delta k)=\sum_{j}\left|\left\langle\psi_{j}(k) \mid \psi_{i}\left(k_{0}\right)\right\rangle\right|^{2} \delta\left(\omega-\omega_{i j}\left(k, k_{0}\right)\right),
$$

with $\omega_{i j}\left(k, k_{0}\right)=\omega_{i}\left(k_{0}\right)-\omega_{j}(k)$ and $\delta k \equiv k-k_{0}$ the perturbation strength. Eq. 1 1 shows that the LDOS is a density of states in which the delta functions are weighed by the overlaps between perturbed and unperturbed states. In addition, the LDOS width gives an estimation of how many perturbed states contribute to an unperturbed one. Besides, it is the Fourier transform of the fidelity amplitude (FA) of the state $\left|\psi_{i}\left(k_{0}\right)\right\rangle$,

$$
\rho_{i}(\omega, \delta k)=\mathcal{F}\left[\left\langle\psi_{i}\left(k_{0}\right)\left|e^{i H(k) t / \hbar} e^{-i H\left(k_{0}\right) t / \hbar}\right| \psi_{i}\left(k_{0}\right)\right\rangle\right] .
$$

Both the FA and its absolute square value, called the Loschmidt echo, are important measures of sensitivity to perturbations and irreversibility of quantum evolutions 2 6].

The LDOS has been considered in many contexts. In a seminal paper, Wigner studied the LDOS in a simple model of banded random matrices [1]. Subsequently, many authors have used the LDOS to characterize the structure of the eigenstates of different random matrix models 7 9]. The LDOS has also been studied in several microscopic systems as for example in a Ce atom 10], in chaotic billiards [11] or a system of a particle that evolves in a smooth Hamiltonian [12]. In addition, the LDOS has been studied to characterize the effect of perturbations in the operation of quantum computers in the presence of static imperfections [13, 14]. It was shown that depending on the characteristics of the system, the LDOS has many regimes as a function of the perturbation strength $\delta k$. However, all the mentioned studies have revealed a region of perturbation strength in which the LDOS has a lorentzian shape, that has been usually called BreitWigner distribution.

A step forward has been recently made in the understanding of the LDOS for chaotic systems [15]. Its relation with the FA has been exploited to develop a semiclassical theory of the LDOS for locally perturbed billiards or maps, that is, when the perturbation is concentrated in a small region of the phase space accessible for the system. It was shown that the LDOS has a Lorentzian shape under very general perturbations of arbitrarily high intensity an a semiclassical expression for its width was derived. This expression only depends on the perturbation, while the properties of the system are taken into account through a uniform measure in phase space. The same results were obtained in a subsequent publication for maps that are globally perturbed but the dynamics was assumed to be completely random [16].

The aim of our study is to test the validity of the semiclassical theory of Ref. [15, 16] in quantum maps when the perturbation is applied in all the phase space and the dynamics of the classical map is not completely random.

* wisniacki@df.uba.ar 
We also consider perturbations that act in an region of the phase space. We study the behavior of the LDOS for maps with different degree of chaoticity and intensity of the perturbation. For this purpose we consider two of the most paradigmatic systems of quantum chaos studies: the perturbed cat map and the Harper map. We show that the semiclassical approximation of the width of the LDOS works very well even for systems with mixed dynamics in which chaos coexist with regular islands. The prediction of Lorenzian shape of the LDOS is fulfilled for highly chaotic maps or when the intensity of the perturbation is big enough.

The paper is organized as follows. In Sec. III we introduce the dynamical systems that we have used for the numerical study, the cat and the Harper maps. In this section,we describe the main characteristics of the classical and quantum dynamics of the maps. Sec. [III is devoted to present the semiclassical theory of the LDOS [15, 16]. The starting point of this theory is a semiclassical approximation of the fidelity amplitude called Dephasing Representation [19]. In Sec. [V] we study the behavior of the LDOS for the systems in several situations and test the validity of the semiclassical theory. We consider various degree of chaoticity and intensities of the perturbation. We also compare the cases ol local and global perturbations. Finally, we conclude with a summary of our results and some final remarks in Sec. V]

\section{SYSTEMS: MAPS ON A TORUS}

An usual procedure to understand a complex behavior is to consider very simple systems in which such a phenomena is observed. The most simple dynamical systems which develops all types of complexity are abstract maps. Due to their simplicity, classical and quantum maps have been very important in the development of classical and quantum chaos [20 22]. Furthermore, many quantum maps have been implemented experimentally in previous studies 23 25].

In this paper we have used maps acting on a torus phase space of area $\mathcal{A}=1$. In particular we have considered the well known cat and Harper maps. These maps possess all the essential ingredients of chaotic and mixed dynamics and are extremely simple from a numerical point of view.

The cat maps are linear automorphisms of the torus that exhibit hard chaos. Anosov's theorem [26] establishes that the cat maps are structurally stable, that is, the orbits of a slightly perturbed map are conjugated to those of the unperturbed map by a homeomorphism. A perturbation of a cat map can be represented by matrices acting on the coordinates

$$
\left[\begin{array}{l}
q^{\prime} \\
p^{\prime}
\end{array}\right]=G\left[\begin{array}{l}
q \\
p
\end{array}\right]+\left[\begin{array}{l}
0 \\
1
\end{array}\right] \epsilon(q, k) \quad(\bmod 1)
$$

where $G$ is a $2 \times 2$ matrix with integer elements choosen that $\operatorname{Tr}(G)>2$ and $\operatorname{det}(G)=1$ since the maps are hyperbolic and conservative. We consider a perturbation

$$
\epsilon(q, k)=(k / 2 \pi)[\cos (2 \pi q)-\cos (4 \pi q)],
$$

with the perturbation strength $k<0.11$ to satisfy the Anosov theorem [26, 27]. To take into account different degrees of chaoticity, in this paper we have considered the following matrices $G$,

$$
G_{1}=\left(\begin{array}{cc}
2 & 1 \\
1 & 1
\end{array}\right), \quad G_{2}=\left(\begin{array}{cc}
80 & 1 \\
6399 & 80
\end{array}\right)
$$

The corresponding Lyapunov exponents, which determine the rate of exponential divergence of classical trajectories are $\lambda_{1} \approx 0.96$ and $\lambda_{2} \approx 5.07$. We note that $\lambda$ is approximately uniform over the whole phase space and nearly independent of $k$ 28].

Perturbed cat maps do not capture all the possible motions of Hamiltonian systems. The most common situation is a mixture of regular islands interspersed by chaotic regions. To consider this general situation the model that we have chosen to study is the Harper map in the unit square [29],

$$
\begin{aligned}
& q^{\prime}=q-k \sin 2 \pi p \quad(\bmod 1), \\
& p^{\prime}=p+k \sin 2 \pi q^{\prime} \quad(\bmod 1),
\end{aligned}
$$

where $k$ is a parameter that controls the behavior of the system. This map can be understood as the stroboscopic version of the flow corresponding to the (kicked) Hamiltonian

$$
H(p, q, t)=-\frac{1}{2 \pi} \cos (2 \pi p)-\frac{k}{2 \pi} \cos (2 \pi q) \sum_{n} \delta(t-n k) .
$$

This is an approximated Hamiltonian for the motion of an an electron in a crystal under the action of an external field.

The Harper map presents a mixed dynamics that depends on the parameter $k$. Fig. 1 1 shows some phase space pictures for this model as an example of the underlying classical dynamics. As can be seen in Fig. 11(left panel), the system presents a mixed dynamics with regions of regularity around the origin and the corners coexisting with chaos, in agreement with the KAM theorem [26]. When the parameter $k=200$ the size of the island are so small that it is not possible to observe without a finer resolution [see Fig. 11 (right panel)].

The quantization on the torus implies that the wave function should be periodic in both position and momentum representation. If in the coordinate and momentum representation the wave function has a period 1 with spacing $1 / N$, it follows that $1=2 \pi \hbar N$. Then, we have a Hilbert space of $N$ dimension for a fixed value of $\hbar$. As $N$ takes increasing values, we reach the semiclassical 


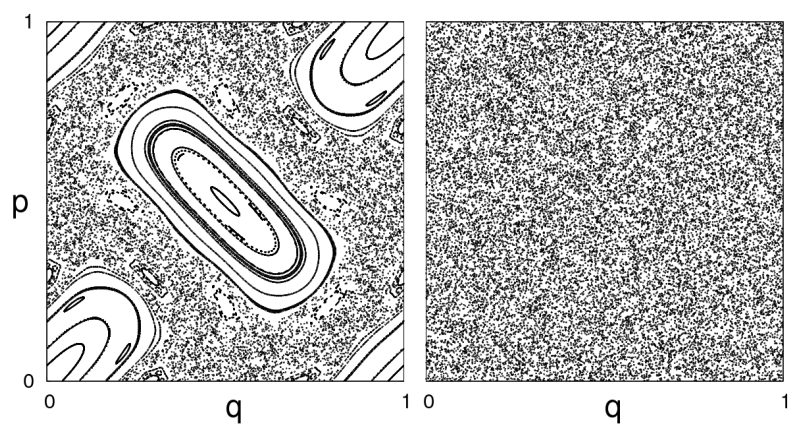

FIG. 1. Classical phase space of the Harper map for $k=0.3$ (left panel) and $k=200$ (right panel). See text for details.

limit. The position basis $\left\{q_{i}\right\}_{i=1}^{N-1}$ (with $\left.q_{i}=i / N\right)$ and momentum basis $\left\{p_{i}\right\}_{i=1}^{N-1}$ (with $\left.p_{i}=i / N\right)$ are related by the discrete Fourier transform. In this setting a quantum map is simply a unitary $U$ acting on an $N$ dimensional Hilbert space and evolution after $n$ steps is given by $U^{n}$.

There is no general method for map quantization. For the perturbed cat map we have considered the quantization based on the classical propagator of Ref. 20, 27]. In this case, the matrix elements of the propagator in the position basis are

$$
\begin{array}{r}
U_{k}^{C}\left(q^{\prime}, q\right)=\sqrt{\frac{N}{i g_{12}}} \exp \left[\frac{i \pi N}{g_{12}}\left(g_{11} q^{2}-2 q^{\prime} q+g_{22} q^{2}\right)\right] \\
\exp \left[\frac{i k N}{2 \pi}\left(\sin (2 \pi q)-\frac{1}{2} \sin (4 \pi q)\right)\right](7)
\end{array}
$$

where $g_{i, j}$ are the elements of the matrix $G$ and we have used $g_{12}=1$.

For the Harper map [29], the matrix elements of evolution operator in the mixed basis of position and momenta are

$$
U_{k}^{H}(q, p)=e^{i N k \cos (2 \pi q)} e^{i N k \cos (2 \pi p)} .
$$

\section{SEMICLASSICAL THEORY OF THE LDOS OF CHAOTIC MAPS}

The LDOS as defined in Eq. 11depends on the characteristics of the state $\left|\psi_{i}\left(k_{0}\right)\right\rangle$. To avoid any dependence with some particular characteristics of this state an average over unperturbed states is performed. Due to the finite number of states in quantum maps, we average over all the Hilbert space. Thus, the averaged LDOS $\rho(\omega, \delta k)$ is

$$
\rho(\omega, \delta k)=\frac{1}{N} \sum_{i=1}^{N} \rho_{i}(\omega, \delta k)
$$

The inverse Fourier transform of Eq. 9, the so called average fidelity amplitude (AFA), is the starting point of the a semiclassical approximation of the LDOS,

$$
\overline{O(t, \delta k)}=\frac{1}{N} \sum_{i}\left\langle\psi_{i}\left(k_{0}\right)\left|e^{i H(k) t / \hbar} e^{-i H_{0}\left(k_{0}\right) t / \hbar}\right| \psi_{i}\left(k_{0}\right\rangle \hat{1} 0\right)
$$

To evaluate Eq. 10] we have used the so called dephasing representation, a semiclassical formulation for fidelity amplitude which avoids the usual trajectory-search problem of the standard semiclassics 19]. One of the forms of the FA obtained using the dephasing representation is

$$
O_{\phi}(t, \delta k)=\int W_{\phi}(q, p) e^{-i \Delta S_{t}(q, p, \delta k) / \hbar} d q d p
$$

where $\Delta S_{t}(q, p, \delta k)$ is the action difference evaluated along the umperturbed orbit starting at $(q, p)$ that evolves at a time $t$ and $W_{\phi}(q, p)$ is the Wigner function of the initial state $|\phi\rangle$. Then,

$$
\overline{O(t, \delta k)}=\int W(q, p) e^{-i \Delta S_{t}(q, p, \delta k) / \hbar} d q d p,
$$

where $W(q, p)=(1 / N) \sum W_{i}(q, p)$, with $W_{i}(q, p)$ being the Wigner function of $\left|\psi_{i}\left(k_{0}\right)\right\rangle$. For chaotic systems, the mean value of the Wigner function for a base of eigenstates is approximately a uniform distribution so $W(q, p)=1 / V$ where $V$ is the volume of the phase space. Therefore,

$$
\overline{O(t, \delta k)}=\frac{1}{V} \int e^{-i \Delta S_{t}(q, p, \delta k) / \hbar} d q d p .
$$

Time is discrete in maps, so from now on we use the integer $n$ to count time steps and $V$ is the area of the phase space that in our case is equal to unity.

In order to solve Eq. 13 for maps we need to assume that trajectories become uncorrelated between two successive hits in the perturbed region. This approximation is valid when the perturbation acts on an infinitesimal portion of phase space [15, 17, 18] or if the unperturbed dynamics of the system is completely random [16].

Here we have considered the second case, the $\lambda \rightarrow \infty$ limit, by assuming that the dynamics is purely random. This evolution is completely stochastic in the sense that there is no correlation for the different times of the evolution. Then, to compute $\overline{O(n, \delta k)}$, we have divided the phase space in $N_{c}$ cells. The probability to jump from cell to any other in phase space is uniform. Therefore it is straightforward to show that the mean FA results

$$
\begin{aligned}
\overline{O(n, \delta k)} & =\sum_{j_{1}} \ldots \sum_{j_{n}} e^{\left[-i\left(\Delta S_{j_{1}}+\ldots+\Delta S_{j_{n}}\right) / \hbar\right]} \\
& =\left(\sum_{j} e^{\left(-i \Delta S_{j} / \hbar\right)}\right)^{n},
\end{aligned}
$$


where $\Delta S_{j p}$ is the action difference evaluated in the cell $j$ at time $p$. The continuous limit is approached when $N_{c} \rightarrow \infty$ resulting in

$$
\overline{O(n, \delta k)}=\left(\int e^{-i \Delta S(q, p, \delta k) / \hbar} d q d p\right)^{n},
$$

where $\Delta S(q, p, \delta k)$ is the action difference after one step of the map.

The exponential decay of Ec. 15] can be rewritten as

$$
\overline{O(n, \delta k)}=e^{-\Gamma n+i \varphi n}
$$

with

$$
\Gamma=-\ln \left(\left|\int e^{-i \Delta S(q, p, \delta k) / \hbar} d q d p\right|\right)
$$

and

$$
\varphi=\arg \left(\int e^{-i \Delta S(q, p, \delta k) / \hbar} d q d p\right) .
$$

We note that $\Gamma$ and $\varphi$ depend on the perturbation strength $\delta k$.

Now, we obtain the semiclassical expression for the average LDOS by the inverse Fourier transform of Eq. 16.

$$
\rho_{s c}(\omega, \delta k)=\mathcal{F}_{[\bar{O}]}^{-1}(\omega, \Gamma, \varphi)=\frac{\Gamma}{\pi\left[(\omega-\varphi)^{2}+\Gamma^{2}\right]} .
$$

The phase $\varphi$ determines the location of the center of the Lorenzian function and $\Gamma$ its width.

Finally, we have to take into account the fact that the spectrum of a map is periodic because of a compact phase space. This periodicity changes the form of the LDOS into a periodized Lorentzian function

$$
\begin{aligned}
\rho_{s c}(\omega, \delta k) & =L^{(p)}(\omega, \Gamma, \varphi) \\
& =\sum_{j=-\infty}^{\infty} \frac{\Gamma}{\pi\left[(\omega-\varphi-2 \pi j)^{2}+\Gamma^{2}\right]} .
\end{aligned}
$$

The same semiclassical expressions for the LDOS were obtained in Ref. [15, 17] when the perturbation acts in a region of the phase space of area $\alpha \rightarrow 0$.

A magnitude that has physical interest is the width $\sigma$ of the LDOS which is a measure of the number of perturbed states that are needed to describe a unperturbed one. Therefore, this quantity offers clear information about the effect of perturbations on on a quantum system. Moreover, the width of the LDOS determines for some regime of the perturbation, the rate of fidelity decay under imperfect motion reversal (the Loschmidt echo). There are different ways of determining this width of a

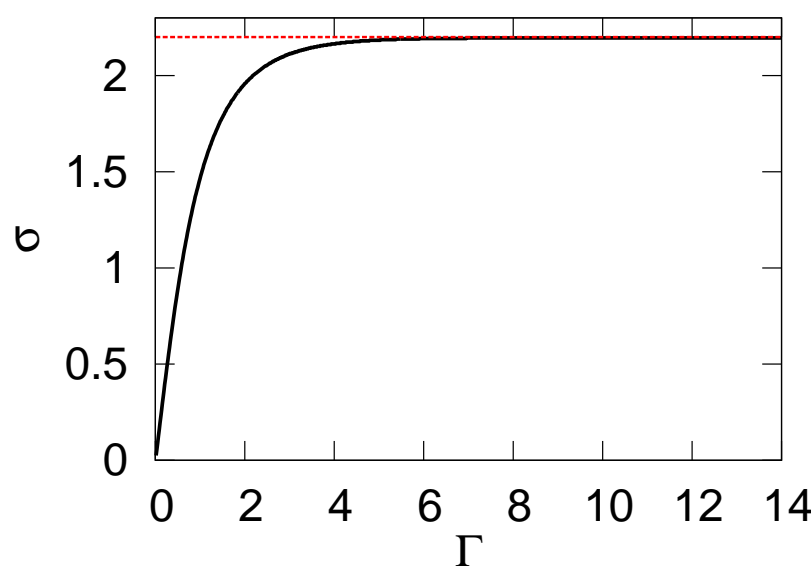

FIG. 2. (Color online) . Witdh $\sigma$ vs. $\Gamma$ for a periodized Lorentzian function [Eq. 20]. The limit of $\sigma$ for $\Gamma \rightarrow \infty$ which corresponds for a constant LDOS is also plotted with red dotted line.

distribution. In our case we are going to take the distance about the average value of the LDOS that contains $70 \%$ of the probability. That is,

$$
\int_{\langle\omega\rangle-\sigma}^{\langle\omega\rangle+\sigma} \rho(\omega, \delta k) d \omega=0.7
$$

where

$$
\langle\omega\rangle=\int_{-\pi}^{\pi} w \rho(\omega, \delta k) d \omega
$$

We show in Fig. 2 the relation between its width $\sigma$ and $\Gamma$ for the periodized Lorentzian function of Eq. 20 .

\section{RESULTS}

The main interest of a semiclassical theory is to describe quantum mechanical quantities using classical information. In this section we show the behavior of the LDOS for the quantum maps presented before and test the validity of the semiclassical approximation of the LDOS described in the previous section. The aim this section is to compare the approximated $\rho_{s c}$ and $\sigma_{s c}$ with the corresponding exact quantum values. The latter are numerically computed by diagonalization of the evolution operators of Eq. 7 and 8

The semiclassical approximation of the LDOS is completely determined by $\Gamma$ and $\varphi$ that are obtained with the calculation of the integral of Eq. 15. To avoid the dependence of the results with the dimension of the Hilbert space $N$ we have considered all the studied quantities as a function of the scaled strength of the perturbation

$$
\chi \equiv\left(k-k_{0}\right) /(2 \pi \hbar)=\delta k N \text {. }
$$




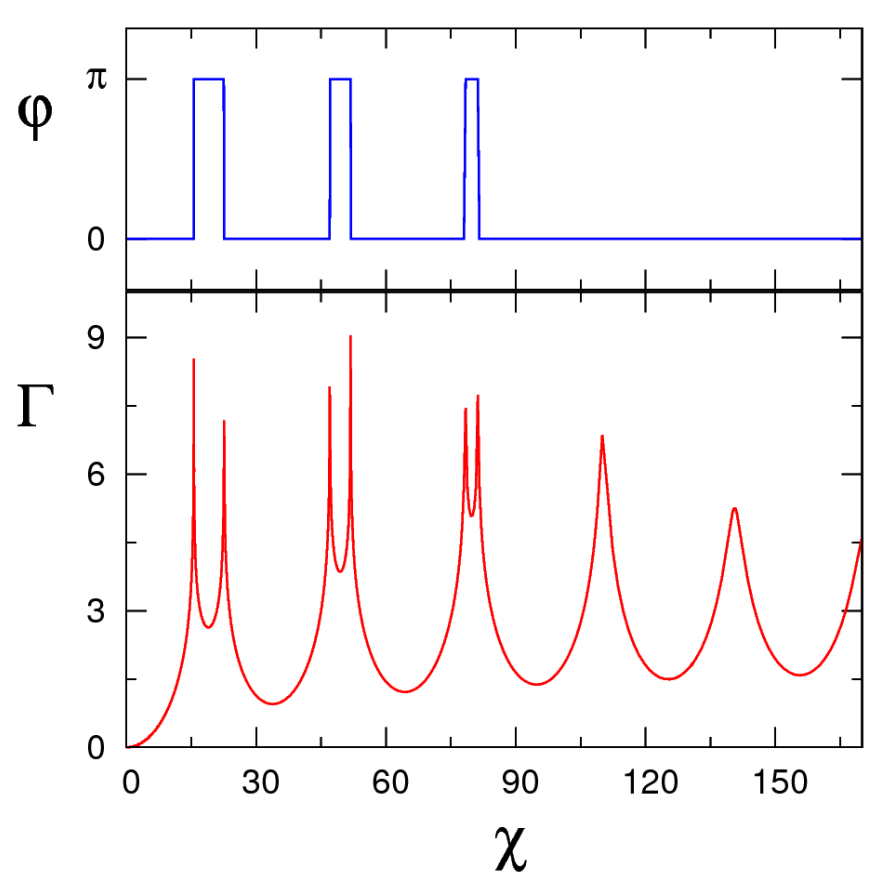

FIG. 3. (Color online) $\Gamma$ and the phase $\varphi$ as a function of the scaled pertubation $\chi$ for the perturbed cat map.

In all the calculations included in this section the number of states of the Hilbert space set as $N=2000$.

\section{A. Peturbed cat map}

The action difference for one iteration of the perturbed cat map described in Sec. II is given by

$$
\Delta S(q, p, \delta k)=\left(\frac{\delta k}{4 \pi^{2}}\right)\left[\sin (2 \pi q)-\frac{1}{2} \sin (4 \pi q)\right] .
$$

Using Eq. 24, 17 and 18 we compute $\Gamma$ and $\varphi$. In Fig. 3 we plot $\Gamma$ and $\varphi$ for the perturbed cat map as a function of the scaled perturbation strength $\chi$. We can see that for perturbation of Eq. 4. $\varphi$ has only two possible values either 0 or $\pi$.

We firstly compare the semiclassical approximation of the width of the LDOS with the corresponding quantum value. For this reason the width of the LDOS has been computed for the cat map using $k_{0}=0.01$ to avoid all the arithmetic peculiarities of the cat map $(k=0)$, which account for the non-generic spectral statistics [30]. In Fig. 4 the width of the LDOS is shown for the cat maps with $G_{1}$ and $G_{2}$. The semiclassical approximation $\sigma_{s c}$, plotted in solid line, works extremely well for both cat maps in the whole range of considered perturbations.

The width of the LDOS $\sigma$ for the cat maps has two clearly different regimes [ Fig. 目 . For small perturbation strength when $\chi \lesssim 10$ it presents a quadratic behavior that is usually called Fermi Golden Rule regime.

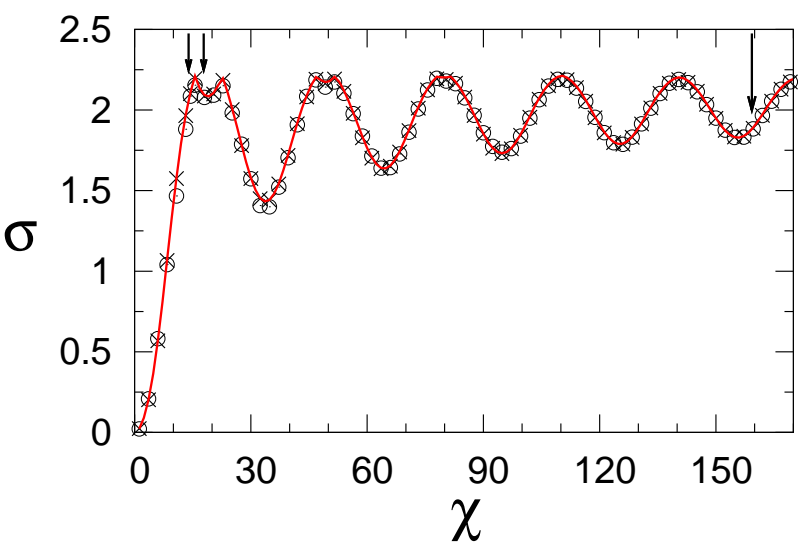

FIG. 4. (Color online) Width $\sigma$ of the LDOS as a function of the scaled perturbation strength $\chi=\left(k-k_{0}\right) N$ for the cat map with $G_{1}(\mathrm{O})$ and $G_{2}(\times)$. The red solid line is the semiclassical approximation of $\sigma$. The number of states of the Hilbert space $N=$ 2000 and $k_{0}=0.01$. We indicate with arrows the perturbations strength of the LDOS displayed in Fig. 5

Conversely for greater strength the width is an oscillating function. In order to understand the behavior of $\sigma_{s c}$ when $\chi \rightarrow \infty$ we have used the stationary phase approximation method to solve the integral Eq. 13 obtaining

$$
\Gamma \rightarrow-\log [1 / \sqrt{\chi}] \quad \text { for } \quad \chi \rightarrow \infty,
$$

therefore the width $\sigma_{s c} \rightarrow 0.7 \pi$ that corresponds to a uniform distribution.

At this point we would like to see how good the semiclassical approximation of the LDOS can describe the complete distribution. We have therefore computde the quantum LDOS for several values of perturbation strength for the cat maps $G_{1}$ and $G_{2}$. In Fig. 5 we compare the LDOS with its semiclassical approximation for perturbations indicated in Fig. 4 with arrows. Fig. 5 (a) corresponds to the most chaotic case $G_{2}$. In the main plot $\chi=159.6$, in the left inset $\chi=14.4$ and in the right inset $\chi=18$. We can see that the semiclassical approximation works very well for all the perturbations, that is, the LDOS is a periodized Lorentzian function indistinctly of the perturbation strength. Left and right inset corresponds to approximately the same width of the distribution but in the left figure $\varphi=0$ and $\varphi=\pi$ for the right so in this case the periodized Lorentzian is centered in $\omega=\pi$. As can be seen in Fig. 3, near $\chi \approx 15$, the phase $\varphi$ has a discontinuity and jumps from 0 to $\pi$, for this reason the center of the LDOS changes from $\omega=0$ to $\omega=\pi$. Similar behavior occurs in the other discontinuities of $\varphi$ near $\chi \approx 50$ and 70 .

In Fig. 5(b) the results for the cat map with the matrix $G_{1}$ are shown. In the figure main panel, we show that for big perturbation strength after the quadratic regime $(\chi=159.6)$ the LDOS is well described by 


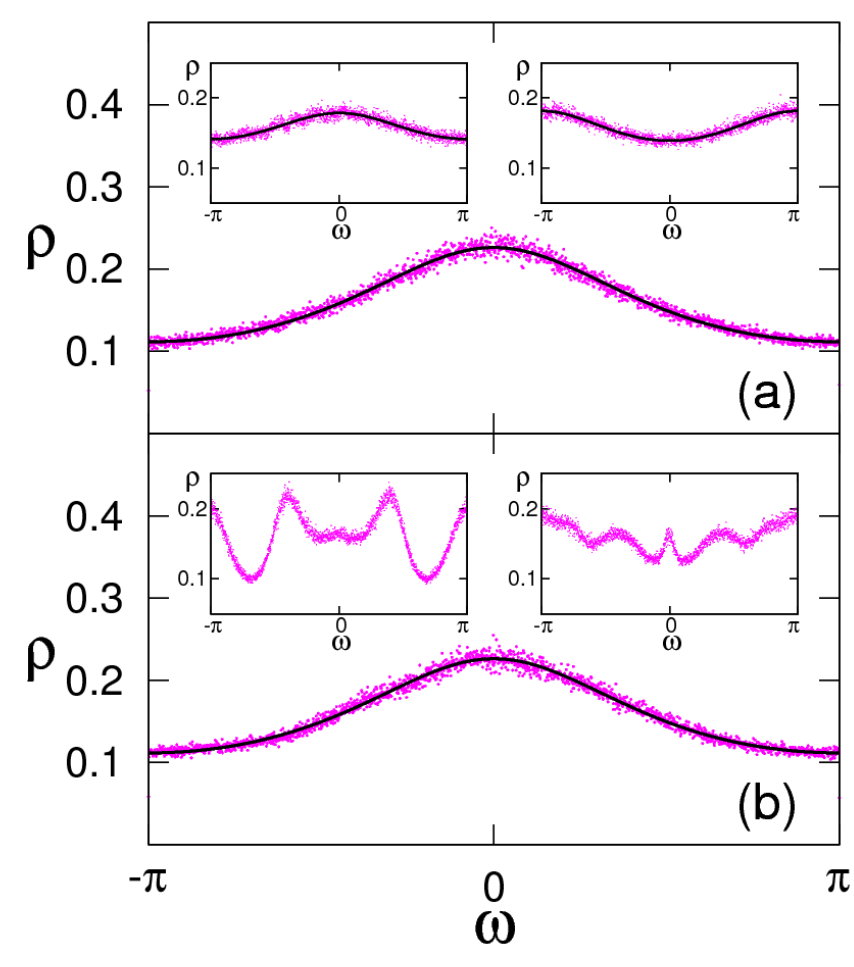

FIG. 5. (Color online) LDOS $\rho$ (points) and its semiclassical approximation (solid line). (a) cat map of Eq. 3 with $G=G_{2}$ for a scaled perturbation strengh $\chi=159.6$ (main plot), $\chi=14.4$ (left inset) and $\chi=18.0$ (right inset). (b) cat map of Eq. 3 with $G=G_{1}$ for $\chi=159.6, \chi=14.4$ (left inset) and $\chi=18.0$ (right inset).

the semiclassical Lorentzian distribution. Conversely for smaller perturbations strength the LDOS does not show a Lorentzian behavior [see inset of Fig. 5(b)]. To understand this behavior we show in Fig. [6 the mean value of the fidelity amplitude $\overline{O(n)}$ for $\chi=14.4$ of both cat maps with $G_{1}(\mathrm{\circ})$ and $G_{2}(\times)$ which corresponds to the inverse Fourier transform of the LDOS plotted in the left inset of Fig. 5(a) and (b). We see that in the case in which the LDOS is not a periodized Lorentzian function the corresponding $\overline{O(n)}$ has a big revival (at $n=4$ ). This kind of behavior, known as survival collapse after which the largest revivals appear, was observed in a spin chain [32] and can be the cause for non-Markovian quantum evolutions [33].

We test now the validity of the semiclassical approximation of the LDOS for local perturbation. For this reason the perturbation is applied in a $q$ strip from $q_{0}=0.25$ to $q_{1}=0.46$ so the area of the perturbed region is $\alpha=\Delta q \Delta p=q_{1}-q_{0}=0.21$. In Fig. 7 we show $\Gamma$ and $\varphi$ as a function of the scaled perturbation strength $\xi$ computed using Eq. 17 and 18. We can see that for this local perturbation $\varphi$ is an oscillating function so the semiclassical approximation of the LDOS is periodized Lorentzian function with an oscillating mean value. In Fig. 7 (top panel) the mean value of the exact LDOS it is also plotted with $(\square)$ showing that the semiclassical $\varphi$

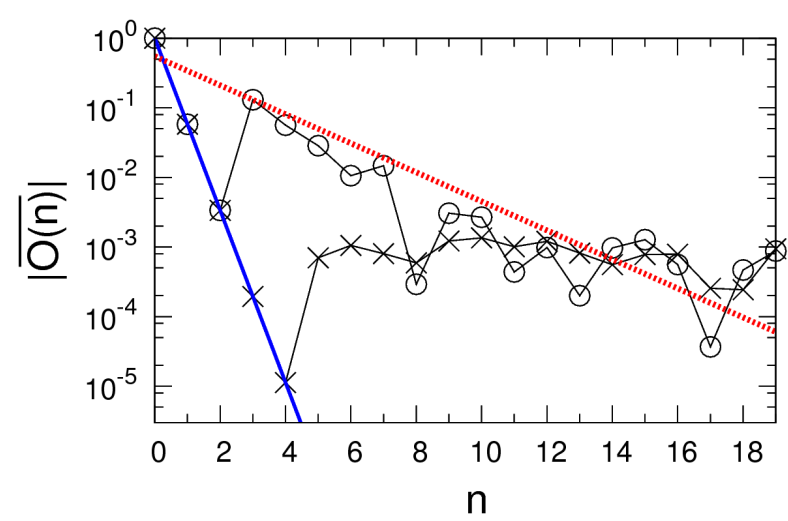

FIG. 6. (Color online) Mean value of the amplitude fidelity $\overline{O(n)}$ for the cat map with $G_{1}(\mathrm{O})$ and $G_{2}(\times)$ with perturbation strength $\chi=14.4$. The exponential decay given by $\exp (-\Gamma n)$ is plotted with solid blue line. We also plot with dotted red line the exponential decay given by $\exp (-\lambda n / 2)$ with $\lambda$ the Lyapunov exponent for the cat map with $G_{1}$.

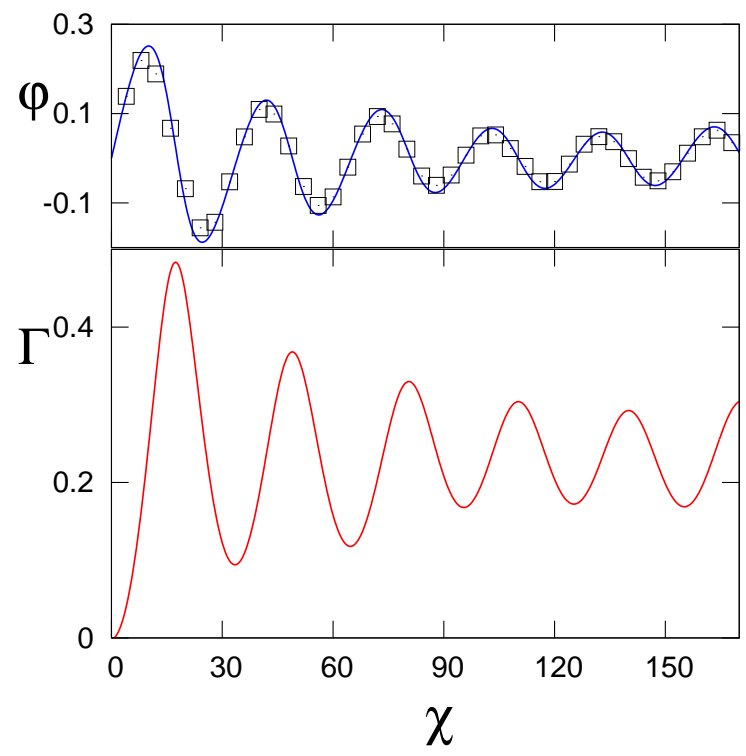

FIG. 7. (Color online) $\Gamma$ and the phase $\varphi$ as a function of the scaled pertubation $\chi$ for the cat map when the perturbation is applied in a $q$ strip from $q_{0}=0.25$ to $q_{1}=0.46$. The mean value of the quantum LDOS is also plotted with $(\square)$.

describe very well this quantity.

The LDOS is also very well approximated by the semiclassical LDOS for all the perturbations strength that we have studied. In Fig. 8 we show the LDOS for in $\chi=8$ when the width grows cuadratically (FGR regime) and for $\chi=28$ when the width shows an oscillating behavior. The semiclassical approximation is plotted with solid line. In the inset of Fig 8 we show the width of the LDOS for this local perturbation and its semiclassical approximation. We can clearly see that the $\sigma_{s c}$ works very well 


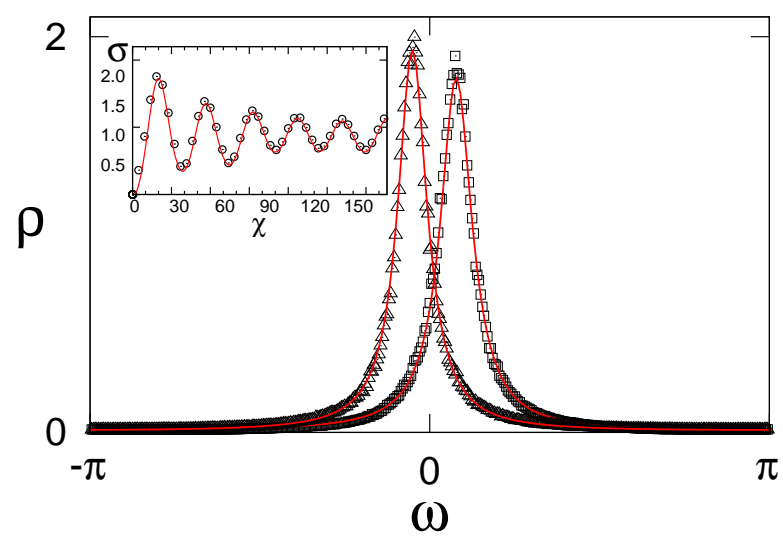

FIG. 8. (Color online) LDOS $\rho$ for a local perturbations. The cat map with $G_{1}$ is perturbed from $q_{0}=0.25$ to $q_{1}=0.46$. The scaled perturbation strength $\chi=8(\triangle)$ and $\chi=28(\square)$. The semiclassical approximation of the LDOS is plotted with red solid line. Inset: Width $\sigma$ of the LDOS as a function of the scaled perturbation strength $\chi(\circ)$ and with red solid line the semiclassical approximation.

for local perturbation. It is noteworthy that all the calculations for local perturbations were done using the map $G_{1}$ showing that when the perturbation is applying is a small region of the phase space less degree of chaoticity is needed for the semiclassical LDOS to be accurate.

\section{B. Harper map}

We have studied the LDOS of the Harper map using the evolution operator of Eq. 8 with $k=k_{0}+\delta k$. The parameter $\delta k$ is the perturbation strength and as we have used for the cat map, the scaled perturbation straight $\chi=\delta k N$. In this case the action difference for one iteration of the Harper map is given by

$$
\Delta S(q, p, \delta k)=\left(\frac{\delta k}{2 \pi}\right)\left[\cos (2 \pi p)+\cos \left(2 \pi q^{\prime}\right)\right]
$$

where $q^{\prime}$ is given by Eq. 5 ,

We have considered as unperturbed system the cases with $k_{0}=0.30$ [mixed dynamics, Fig. 1(left panel)] and $k_{0}=200$ [chaotic dynamics, Fig. 1(right panel)]. Using Eq. 17, 18 and 20 we compute $\Gamma, \varphi$ and the corresponding semiclassical approximation of the LDOS. In Fig. 9 we show $\Gamma$ as a function of the scaled perturbation strength $\chi$. For the action difference of the Harper map [Eq. 25] we have obtained that $\varphi=0$.

In Fig. 10 we show the width of the LDOS for the Harper map and the corresponding semiclassical approximation. When the dynamics of the Harper map is completely chaotic, the semiclassical $\sigma_{s c}$ works well as expected. Surprisingly, the semiclassical approximation works reasonably well even for mixed dynamics. This

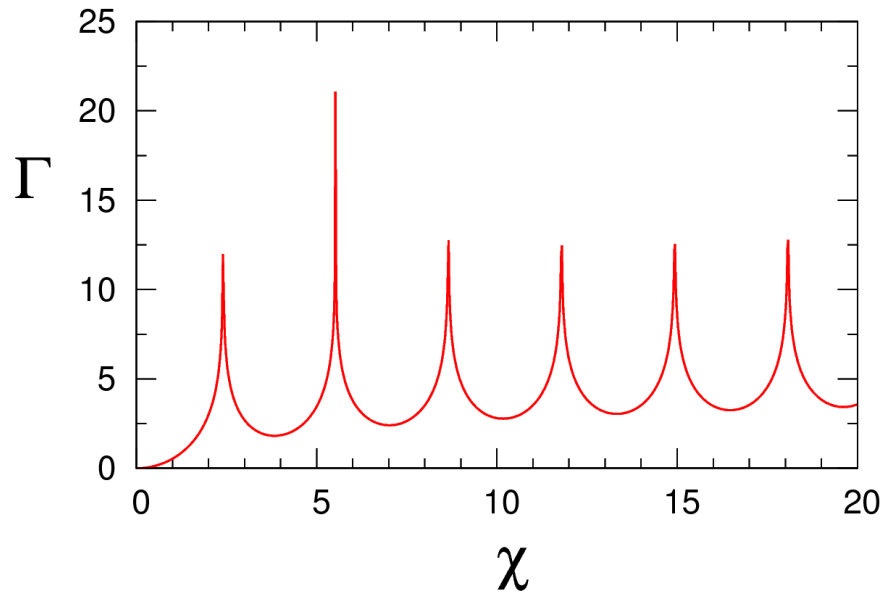

FIG. 9. (Color online) $\Gamma$ as a function of the scaled pertubation strength $\chi$ for the Harper map.

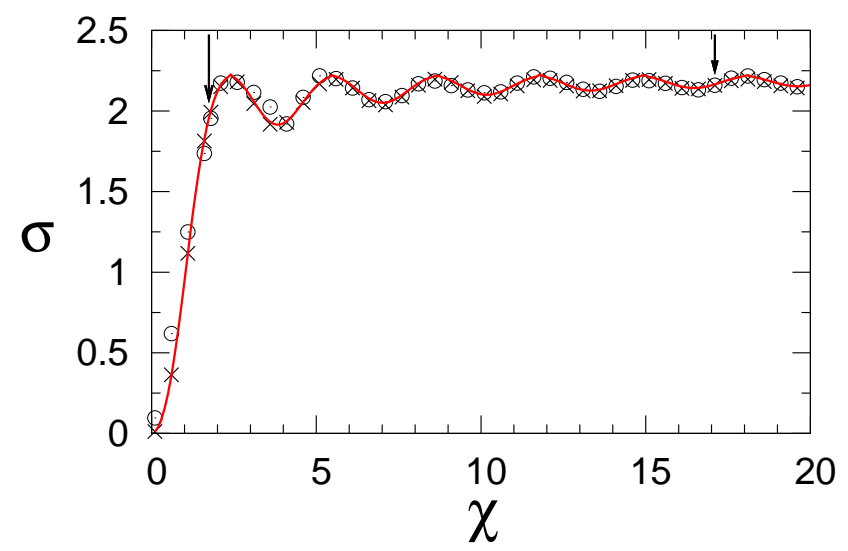

FIG. 10. (Color online) Width $\sigma$ of the LDOS as a function of the scaled perturbation strength $\chi$ for the Harper map with $k_{0}=0.3(\mathrm{O})$ and $k_{0}=200(\times)$. In red line it is plotted the semiclassical approximation $\sigma_{s c}$. We indicate with arrows the perturbations strength of the LDOS displayed in Fig. 11

agreement is more noticeable for bigger $\chi$. The explanation of this unexpected behavior is as follows. Eq. 15] is exact for one time step $(n=1)$ and if the perturbation strength is big enough the fidelity amplitude decays in this short time. Therefore, this short time decay gives the width of the Fourier transform which is the LDOS.

In Fig. 11 we show the LDOS for the Harper map. Although the semiclassical width of the LDOS $\sigma_{s c}$ works well for mixed dynamics, the complete distribution is not well reproduced by a periodized Lorentzian distribution. This is shown in the inset of Fig. 11 (b) for the Harper map with $k_{0}=0.3$ and $\chi=1.7$. If the perturbation strength is bigger [Fig. 11 (a) (main plot)] the semiclassical theory works reasonably well but the quantum LDOS is a more fluctuating function than the chaotic 


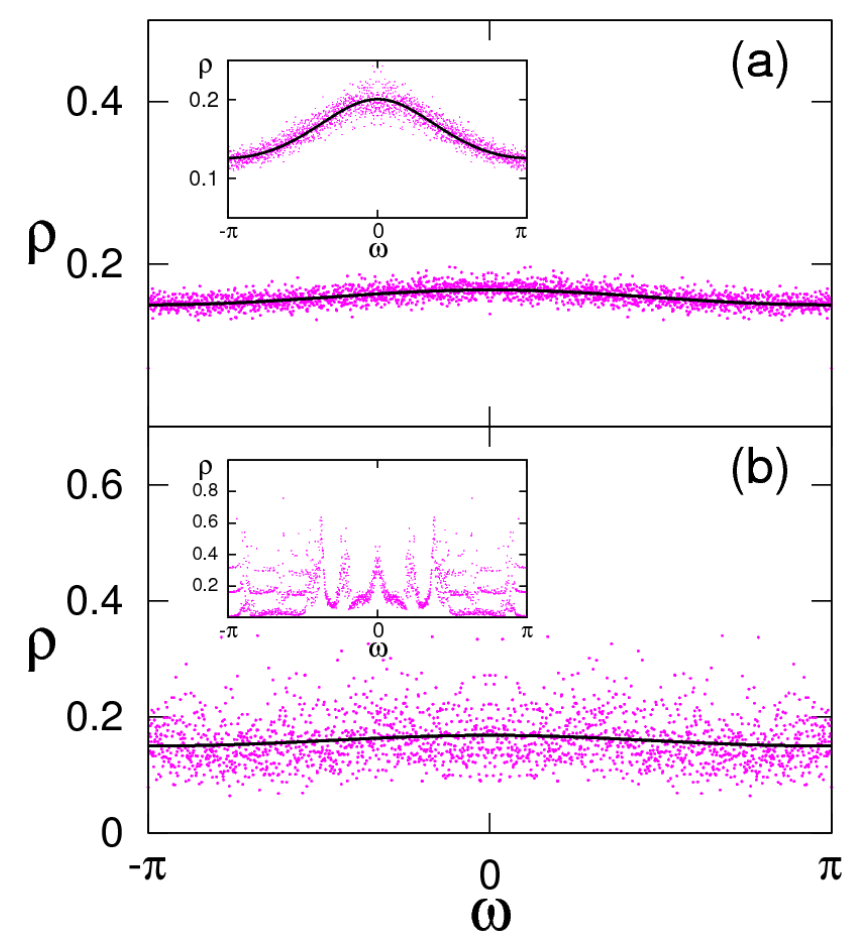

FIG. 11. (Color online) $\rho$ (points) and its semiclassical approximation $\rho_{s c}$ (solid line) for the Harper map. (a) $k_{0}=200$. In the main plot $\chi=17$ and in the inset $\chi=1.8$. In both plot it is seen that the semiclassical LDOS describes the full quantum result. (b) $k_{0}=0.3$ (mixed dynamics). In the main plot $\chi=17$ and in the inset $\chi=1.8$.

case [see Fig. 11 (a)]. As expected, the semiclassical theory works well for the case of $k_{0}=200$ in which the Harper map is fully chaotic (Fig. 11 (a)).

\section{CONCLUSIONS}

The reaction of a system to perturbations is a fundamental problem in quantum mechanics. In this paper we have made a detailed analysis of the response to perturbations of the simplest quantum systems, which can have complex classical dynamics. For this reason, we have studied the LDOS in the perturbed cat map, a completely chaotic system and the Harper map which has mixed dynamics. Our fundamental goal was to discuss the validity of a semiclassical theory of LDOS that has been recently developed [15, 16]. This theory is based on the relation of the LDOS with the fidelity amplitude, a measure of irreversibility and sensitivity to perturbations of quantum systems. Furthermore, it uses the dephasing representation of the fidelity amplitude, a semiclassical formulation that avoids the usual problems of semiclassical theories. The main assumption of the semiclassical theory of LDOS is that the trajectories get uncorrelated after one step of the map. This condition is fullfiled if the dynamics is completely random or when the perturbation is applied in a infinitesimal region of the phase space. Due to the fact that these conditions are not achieved in dynamical system, we tested the validity of such a semiclassical theory of the LDOS.

We have analyzed various situations: local and global perturbations and also we have varied the degree of chaoticity. We show that the LDOS is very well described by its semiclassical expression when the map is highly chaotic, either if the perturbation is localized in phase space, or when the perturbation strength is big enough . We remark that in these cases the semiclassical LDOS completely reproduces the quantum version without any fiting parameters. We have studied the case of mixed dynamics and surprisingly enough our results show that the semiclassical width of the LDOS describes the full quantum version even in this case.

We would like to highlite that our results could be of importance in the study of the LDOS of billiards. Indeed, the behavior of a billiard system has many resemblances with maps. For example, the classical dynamics of a billiard can be described by a map on the boundary. Quantum billiards are realistic systems that can be constructed in experimental setups of several nature. In fact, there are cavities of microwave, acoustic or optical wave. The semiclassical approximation of the width of the LDOS has been successfully applied in billiard that has been perturbed both locally [18, 31] and globally [15]. However, in these works the behavior of the whole distribution was not properly discussed. Further insight on the LDOS of this systems will be part of future studies.

\section{ACKNOWLEDGEMENTS}

The authors acknowledge the support from CONICET (PIP-6137), UBACyT (X237, 20020100100741, 20020100100483) and ANPCyT (1556). We would like to thank Ignacio García Mata for useful discussions.
[1] E. P. Wigner, Ann Math 62548 (1955). ibid 65203 (1957)

[2] R. A. Jalabert and H. M. Pastawski, Phys. Rev. Lett. 86 2490 (2001).

[3] T. Gorin, T. Prosen, T. H. Seligman, M. Z̆nidarič , Phys. Rep. 43533 (2006).
[4] Ph. Jacquod and C. Petitjean, Adv. Phys. 5867 (2009).

[5] D. A. Wisniacki, D.Cohen, Phys. Rev. E 66046209 (2002).

[6] A. Goussev, R. Jalabert, H. Pastawski and D. A. Wisniacki, unpublished. 
[7] Y. V. Fyodorov, O. A. Chubykalo, F. M. Izrailev and G. Casati, Phys. Rev. Lett. 761603 (1996).

[8] G. Casati, B. V. Chirikov, I. Guarneri, and M. Izrailev, Phys. Lett. A 223433 (1996).

[9] Ph. Jacquod and D. L. Shepelyansky, Phys. Rev. Lett. 753501 (1995).

[10] V. V. Flambaum, A. A. Gribakina, G. F. Gribakin, and M. G. Kozlov, Phys. Rev. A 50, 267 (1994).

[11] D. Cohen and E.J. Heller, Phys. Rev. Lett. 84, 2841 (2000).

[12] D. Cohen and T. Kottos, Phys. Rev. Lett. E 63036203 (2001).

[13] B. Georgeot and D. L. Shepelyansky, Phys.Rev. E 62 6366 (2000).

[14] G. Benenti, Giulio Casati, Simone Montangero, and Dima L. Shepelyansky, Eur. Phys. J. D 20, 293 (2002).

[15] D. A. Wisniacki, N. Ares and E. G. Vergini, Phys. Rev. Lett. 104254101 (2010).

[16] I García-Mata, R. O. Vallejos and D. A. Wisniacki, New J. Phys. 13103040 (2011).

[17] I García-Mata, and D. A. Wisniacki, J. Phys. A. Math. Theor. 44315101 (2011).

[18] A.Goussev, D. Waltner, K. Richter, and R. A. Jalabert, New J. Phys. 10, 093010 (2008).

[19] J. Vanícek, Phys. Rev. E 70 055201(R) (2004). Ibid 73 046204 (2006).
[20] J. H. Hannay and M. V. Berry, Physica D 1267 (1980).

[21] N. Balazs and A. Voros Europhys. Lett. 41089 (1987).

[22] J. P. Keating, Nonlinearity 4309 (1991).

[23] F. L. Moore,J. C. Robinson,C. F. Bharucha,B. Sundaram and M. G. Raizen Phys.Rev.Lett. 754598 (1995).

[24] Y. S. Weinstein,S. Lloyd ,J. Emerson and D. G. Cory Phys. Rev. Lett. 89157902 (2002).

[25] J. Chabé,G. Lemarié, B. Grémaud,D. Delande,P. Szriftgiser and J. C. Garreau Phys.Rev.Lett. 101255702 (2008).

[26] V. I. Arnold, Geometrical Methods in the Theory of Ordinary Differential Equations, Springer-Verlag (1988).

[27] M. B. de Matos and A. M. Ozorio de Almeida, Ann. Phys. 23746 (1995).

[28] N. Ares and D. A. Wisniacki, Phys. Rev. E 80046216 (2009).

[29] P. Leboeuf, J. Kurchan, M. Feingold, and D. P. Arovas, Phys. Rev. Lett. 65, 3076 (1990).

[30] J. P. Keating and F. Mezzadri, Nonlinearity 13, 747 (2000).

[31] B. Köber, U. Kuhl, H. J. Stöckmann, A. Goussev, and K. Richter, Phys. Rev. E 83, 016214 (2011)

[32] E. R. Fiori and H. Pastawski, Chem. Phys. Lett. 420, 35 (2006).

[33] I García-Mata, C. Pineda and D. A. Wisniacki, arXiv:1204.3614 